\title{
A business case for risk-informed bridge monitoring using the value of information
}

\author{
Manu Sasidharan ${ }^{1,2}$, Ajith Kumar Parlikad ${ }^{1,2}$, Jennifer Schooling ${ }^{2}$ \\ ${ }^{1}$ Institute for Manufacturing, University of Cambridge, Cambridge, CB3 OFS, UK \\ ${ }^{2}$ Centre for Smart Infrastructure and Construction, University of Cambridge, CB3 OFA, UK
}

Contact: $\underline{\text { mp979@cam.ac.uk }}$

\begin{abstract}
While the benefits of monitoring bridges are fairly understood, securing the resources necessary for their development and installation is often difficult. The premise of the article is that infrastructure owners will take action to provide those resources when they can see value accruing to them; therefore narratives around value generation and business cases are required to motivate and support that action. This paper explores the application of the value of information methodology to the challenge of developing a business case for bridge monitoring using an illustrative case study on a simulacrum of a road bridge in the UK. To this end, this paper presents a systematic approach for setting risk-informed bridge monitoring strategies while considering the reliability and accuracy of data capture offered by different monitoring techniques and the gains to be made downstream if it contributes to setting timely maintenance strategies.
\end{abstract}

Keywords: structural health monitoring; condition monitoring; bridge; value of information; life cycle costs; decision making; asset management;

\section{Introduction}

Infrastructure asset managers face the challenge to manage the ageing and deteriorating bridge portfolio as disruption to their operation or failure can have significant effects beyond the damage to the physical infrastructure, including undesirable health and safety, economic, environmental and political consequences. They must optimally allocate scarce resources among competing objectives and assets within their network. Even if they restrict their attention to devote to the mitigation of a particular risk (e.g. scour), it is not easy to determine how to rationally distribute the available resources. To assess and maintain the bridges effectively, quality information about their condition is essential.

Structural health monitoring (SHM) is becoming a standard feature of infrastructure assets across the world. Many bridges in the UK have or have had
SHM systems installed; for example, Humber bridge, Nine wells bridge, Ferriby road bridge and Hammersmith flyover (1). While there has been significant research $(2,3)$ that discusses how to deploy SHM systems and obtain data, there is a lack of a business case for monitoring as the risks of bridge failure and associated costs are not well understood, and there are no systematic frameworks for addressing the same (4). In other words, there is a gap when it comes to aiding the decisions on if a bridge needs to be monitored and to what level.

While rehabilitation or repairing interventions are effective in reducing the structural vulnerability of bridges, bridge monitoring, on the other hand, do not reduce the vulnerability but provide information about its state. A monitoring system is useful only if the obtained information influences decisions on the operation and maintenance of the bridge (1). The value of the monitoring system depends on the frequency of the events (e.g. 


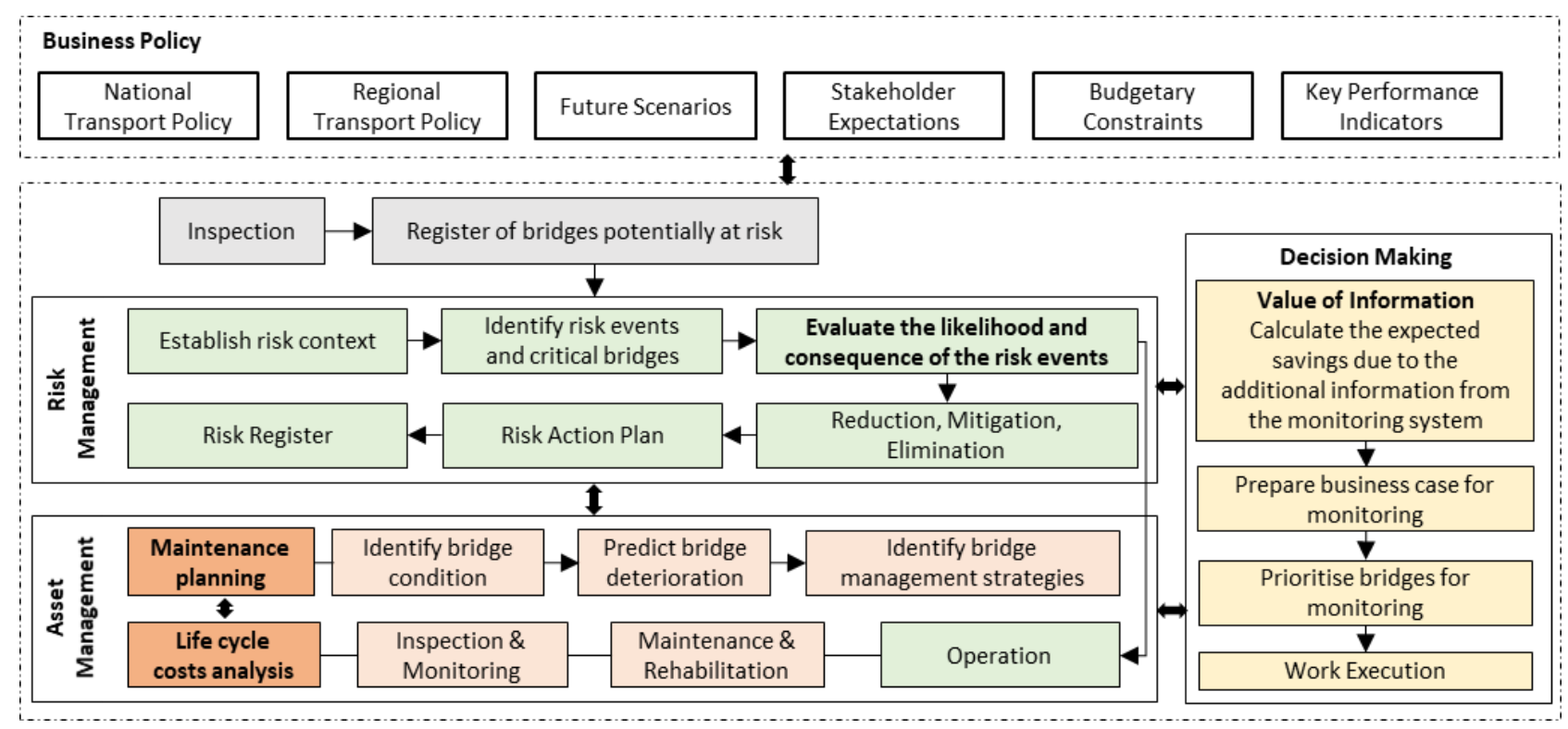

Figure 1 Theoretical framework for risk-informed bridge monitoring at project level

flooding) and defects (e.g. scour), of their consequences, and the quality of the information provided by the monitoring devices. While the reduction in uncertainty when gathering information can be used as a metric for ranking the effectiveness of a monitoring system, it is not sufficient to guide the decision on whether to monitor the bridge or not, as it provides no clue on how this reduced uncertainty improves the decision-making process (5).

The concept of the value of information (Vol) provides a rational means by which the problem of 'to monitor a bridge or not?' can be recast into a formal economic decision framework. Vol measures the difference between the expected benefit (or loss) with and without the information provided by the monitoring system. Such an approach has been previously employed to inform inspection frequencies and maintenance strategies for bridges (6-13). However, despite the significance of the macroeconomic costs of bridge closure or failure, they fail to consider them within the decision making (14). In this paper, a riskinformed framework for assessing the benefit of installing a monitoring system as a decision support tool for the management of bridges while dealing with uncertainties at an individual/project level is proposed.

\section{Theoretical framework}

Given the value of bridges within the transport system, it is important to be able to predict the consequence of closures or failures for each bridge across the system. The conceptual framework proposed within this paper (see Figure 1) shows how asset and risk management should not be separate and independent of the transport authority's business policy but could be a means of interpreting corporate goals in the context of the physical infrastructure asset and its associated impacts to give a clearer focus for the decision making at the individual bridge level. Risk management supports the approach adopted for making decisions through the asset management planning process. A common approach is to commence the risk identification at a high level to obtain an assessment for the level of overall risk exposure. This may then be followed by a detailed assessment of more specific risks where critical assets, critical failure modes and high-risk areas can be defined and analysed in greater detail for their likelihood of occurrence and consequence (15). While the likelihood of a defect occurring is governed by different contributing factors (environmental, structural, geological etc), the magnitude of the consequences is often mediated by the macroeconomic costs or impacts of related bridge closure or failure. While considering the 
macroeconomic impacts or costs of bridge disruption or failure within the decision making, there are some challenges concerning the lack of data associated with costs, impacts, benefits, the spread of defects and bridge foundations, giving rise to uncertainties. Risk assessment techniques such as Monte Carlo, Bayesian, and Petri nets are recommended to deal with such uncertainties [2].

By predicting the condition of the individual bridges and the associated life cycle costs, the operators are then able to identify critical assets that need prioritisation of investments and resources. Such an approach would also aid in making a business case for investing in predictive and preventive asset management regimes. This would translate into the task at hand - to inform the decision-making module on whether to install a monitoring system or not. By employing the Vol concept, the need to monitor and the resulting value in monitoring can be estimated from an economic perspective. If the $\mathrm{Vol}$ is greater than the added cost of obtaining the information (the cost of the monitoring), then it is justified. Thus the proposed risk-informed bridge monitoring framework could be employed by senior managers and decision-makers to identify cost-effective and value-based monitoring strategies for a bridge of a given level of risk of failure.

\subsection{Life cycle analysis}

The life cycle costs (LCC) analysis module takes information from the maintenance planning module to determine the associated costs. The condition assessment and the deterioration estimation highlights the bridges or elements within the network where maintenance intervention is (and will be) required. Different techniques are used to predict the bridge condition namely, Markov chains (16), Petri-net (17), analytical hierarchy process (18), Monte Carlo simulations (19), Bayesian (20) and normal regressions (21). The frequency of routine or preventive maintenance of bridges is based on factors including environmental, operational, design configuration, budgets which are captured within the risk levels assigned to each bridge on the network by the infrastructure owner.
The LCC $(\hat{C})$ under uncertainty of a bridge assessed to be of risk level, $R L$, may be calculated as the sum of inspection and maintenance costs $\left(\widehat{\mathrm{C}}_{\mathrm{M}_{n}}\right)$ and the associated operational impacts $\left(\widehat{C}_{\mathrm{O}_{n}}\right)$ during the year, $n$, using Eqn. (1)

$\hat{C}=\sum_{n=0}^{N} \frac{\widehat{\mathrm{C}}_{\mathrm{M}_{(R L) n}}+\widehat{\mathrm{C}}_{\mathrm{O}_{(R L) n}}}{(1+\hat{\mathrm{r}})^{n}}$

$N$ is the lifespan of the bridge or the analysis period and $r$ is the discount rate. The different cost elements that contribute to the estimation of LCC are calculated using Eqns. (2-3).

\subsubsection{Inspection and maintenance}

The structural condition of the bridge is assessed during periodic inspections. The maintenance thresholds dictated by standards triggers interventions. There are four intervention categories considered for a particular defect namely servicing, minor repair, major repair and replacement. Servicing includes activities that protect the bridge from the source that drives the deterioration process (e.g. cleaning the debris from the waterways, waterproofing etc.). Minor and major repairs are carried out to restore the condition of the bridge/component to a safe and acceptable condition as per standards. Complete replacement of a component or the whole bridge might be required if they are in very poor condition. The direct costs of inspection and maintenance are associated with the required workforce, materials, machinery and scheduling. The total direct discounted costs associated with the inspection and maintenance of bridges during the year, $n$, are calculated using as Eqn. (2) depending upon their frequency, $u$, and deployment of required equipment type, $q$.

$\widehat{\mathrm{C}}_{\mathrm{M}_{n}}=\sum_{u=1}^{U} \sum_{q=1}^{Q}\left(\widehat{\mathrm{C}}_{\mathrm{INS}_{n}}+\widehat{\mathrm{C}}_{\mathrm{SER}_{n}}+\widehat{\mathrm{C}}_{\mathrm{MinR}_{n}}+\right.$

$\left.\widehat{\mathrm{C}}_{\mathrm{MajR}_{n}}+\widehat{\mathrm{C}}_{\mathrm{RM}_{n}}+\widehat{\mathrm{C}}_{\mathrm{REP}_{n}}\right)$

\subsubsection{Operational impacts}

Partial or full-bridge closures are triggered while carrying out inspection, maintenance and replacement. Such closures or failures result in significant disruptions and macro-economic impacts, often beyond the direct costs of the activity; such as costs associated with health and safety $\left(\widehat{\mathrm{C}}_{\mathrm{HS}_{(R L)_{n}}}\right)$, traffic management and user 
costs due to re-routing of traffic $\left(\widehat{\mathrm{C}}_{\mathrm{RR}_{(R L) n}}\right)$, environmental impacts $\left(\widehat{C}_{\mathrm{ENV}_{(R L) n}}\right)$, damage to third party property and goods $\left(\widehat{\mathrm{C}}_{\mathrm{TPD}_{(R L) n}}\right)$, and reputational damage $\left(\widehat{\mathrm{C}}_{\mathrm{RPD}_{(R L) n}}\right)(22)$. To this end, the operational impacts associated with the disruption of a bridge of given risk level, $R L$, during the year, $n$, can be using Eqn. (3)

$$
\begin{aligned}
& \widehat{\mathrm{C}}_{\mathrm{O}_{n}}=\widehat{\mathrm{C}}_{\mathrm{HS}_{(R L) n}}+\widehat{\mathrm{C}}_{\mathrm{RR}_{(R L) n}}+\widehat{\mathrm{C}}_{\mathrm{ENV}_{(R L) n}}+ \\
& \widehat{\mathrm{C}}_{\mathrm{TPD}_{(R L) n}}+\widehat{\mathrm{C}}_{\mathrm{RPD}_{(R L) n}}
\end{aligned}
$$

The individual cost elements are calculated using Eqns. (4-8) as described below.

The costs associated with the injuries and fatalities following the failure of a bridge is estimated using Eqn. (4)

$\widehat{\mathrm{C}}_{\mathrm{HS}_{(R L) n}}=\left(\hat{A}_{I n} * \widehat{W T P}\right)+\left(\hat{A}_{F n} * \widehat{V O L}\right)$

where, $\hat{A}_{I n}$ and $\hat{A}_{F n}$ are the average number of non-fatal injuries and fatalities respectively; $\widehat{V O L}$ is the average economic value for a person's life and $\widehat{W T P}$ is the average economic value that a person is willing to pay for health services.

The costs associated with traffic management $\left(\widehat{\mathrm{C}}_{\mathrm{TM}_{n}}\right)$, increase in vehicle operating costs $\left(\Delta \widehat{\mathrm{VoC}}_{\mathrm{TM}_{n}}\right)$ and delays due to the re-routing during the partial or full closure are captured using Eqn. (5)

$$
\begin{aligned}
& \widehat{\mathrm{C}}_{\mathrm{RR}_{(R L) n}}=\widehat{\mathrm{C}}_{\mathrm{TM}_{n}}+\widehat{\mathrm{N}}_{\mathrm{DJ}_{n}} *\left[\left(\widehat{\mathrm{C}}_{\mathrm{AD}_{n}} * \widehat{\mathrm{T}}_{\mathrm{AD}_{n}}\right)+\right. \\
& \left.\left(\Delta \mathrm{VOC}_{\mathrm{TM}_{n}}\right)\right]
\end{aligned}
$$

The collapse of bridges causes debris and spillages that contaminate the watershed which can be harmful to human health and can harm or kill wildlife. The failure can also release excessive dust and suspended particulates which in turn impacts the surrounding air quality. Such environmental impacts are estimated using Eqn. (6)

$\widehat{\mathrm{C}}_{\mathrm{ENV}_{(R L) n}}=\sum_{p=1}^{P} \widehat{\mathrm{Q}}_{p_{n}} * \widehat{\mathrm{C}}_{\mathrm{p}_{n}}$

where $\widehat{\mathrm{Q}}_{p_{n}}$ is the quantity and $\widehat{\mathrm{C}}_{\mathrm{p}_{n}}$ is the average monetary value of pollutants, $p$, released due to bridge collapse in the year, $\mathrm{n}$.

In the event of a bridge collapse in the given year, $n$, the operator could be sued and might become liable for damages to agricultural land $\left(\widehat{C}_{A L_{n}}\right)$, private properties and goods $\left(\widehat{\mathrm{C}}_{\mathrm{PVT}_{n}}\right)$ and forestry
$\left(\widehat{\mathrm{C}}_{\mathrm{FOR}_{n}}\right)$; fines levied by government agencies $\left(\widehat{\mathrm{C}}_{\mathrm{GOV}_{n}}\right)$ and legal costs $\left(\widehat{\mathrm{C}}_{\mathrm{LEG}_{n}}\right)$. These costs may be estimated using Eqn. (7)

$\widehat{\mathrm{C}}_{\mathrm{TDP}_{(R L) n}}=\widehat{\mathrm{C}}_{A L_{n}}+\widehat{\mathrm{C}}_{\mathrm{PVT}_{n}}+\widehat{\mathrm{C}}_{\mathrm{FOR}_{n}}+\widehat{\mathrm{C}}_{\mathrm{GOV}_{n}}+$

$\widehat{\mathrm{C}}_{\mathrm{LEG}_{n}}$

The financial loss of accidents includes the indirect costs relating to the reputational damage for the operator resulting in loss of consumer and investor confidence that may follow an accident, particularly when the firm is found to be negligent or liable. This can be estimated using Eqn. (8)

$\widehat{\mathrm{C}}_{\mathrm{RPD}_{(R L)_{n}}}=\widehat{\mathrm{MV}}_{a_{n}}-\widehat{\mathrm{MV}}_{\mathrm{b}_{n}}$

where $\widehat{\mathrm{MV}}_{a_{n}}$ and $\widehat{\mathrm{MV}}_{\mathrm{b}_{n}}$ is the market value of the operator following the disruption and before respectively.

\subsection{Decision making}

The bridge infrastructure management process, including maintaining, repairing and operating of a bridge is modelled as a sequential decision making problem (23), where the manager infers and predicts the bridge's condition that evolves due to ageing and deterioration, and takes periodic action with the overall goal of maximising the value of the asset. The decision making within the proposed risk-informed approach to bridge monitoring shown in Figure 1 is modelled as a typical decision tree (see Figure 2). Firstly, a decision on whether or not to obtain more knowledge (e.g. install a monitoring system), and secondly a decision on an action (e.g. carry out maintenance) based on the knowledge is evaluated. The outcomes of these decisions are based on the probability of the existence of the defect, accuracy of its detection by a given monitoring system and the effectiveness of the maintenance interventions based on the knowledge (or the lack of it). The decision problem can be solved by evaluating the costs associated with each branch in the decision tree to identify the one with the greatest value.

\subsubsection{Value of information}

Vol is a utility-based metrics related to decision making under uncertainty, and it measures the expected benefit due to the availability of information. For the task in hand, Vol analysis can 


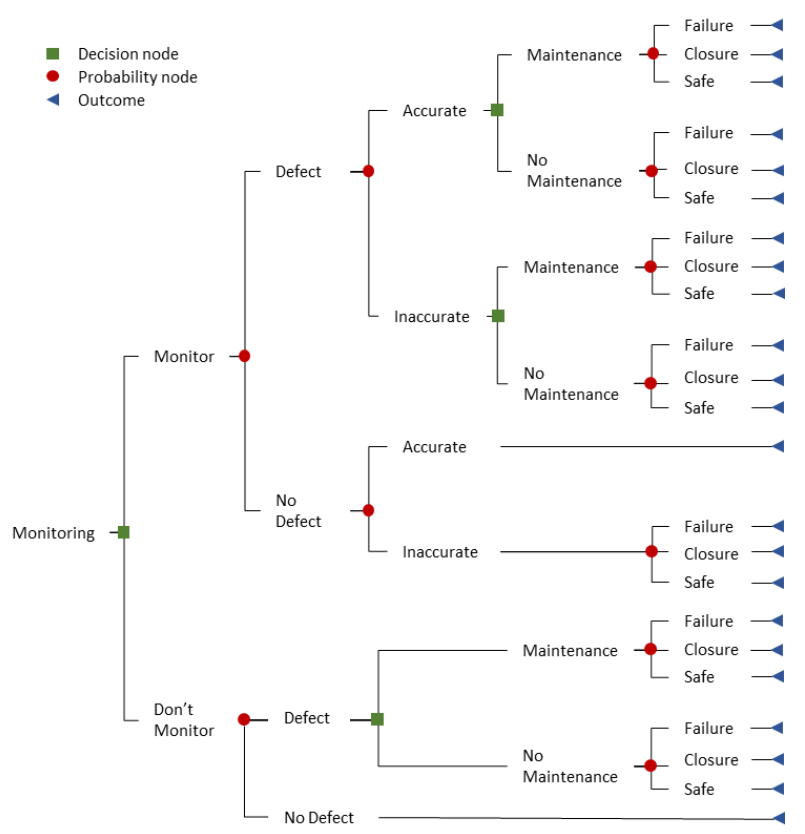

Figure 2 Bridge monitoring decision tree

be performed by taking into account five different events, namely the decision to monitor, existence of the defect, accuracy of defect detection, maintenance and outcome. All the stated events have a probability of occurrence and costs related to their outcomes. The costs of monitoring and maintenance depend on various parameters, such as type of monitoring system, type of repair, operational impacts etc. Thus, the costs of monitoring and maintenance are considered to be relative to each other (7). The Net Life-Cycle Vol ( $\widehat{\mathrm{NVOI}}_{\mathrm{C}}$ ) can be defined as shown in Eqn. (9) where $\hat{C}$ is the LCC of the bridge without the monitoring system (computed by Eqn. (1)) and $\hat{C}^{*}$ is the LCC with the monitoring system.

$\widehat{\mathrm{NVOI}}_{\mathrm{C}}=\hat{C}-\hat{C}^{*}$

where a higher $\widehat{\mathrm{NVOI}}_{\mathrm{LCC}}$ means there is more Vol in monitoring the bridge than otherwise. In the case of applying the proposed approach to decide whether to monitor the bridge or not, we compute the Vol by comparing three outcomes or end state for the bridge: safe, partially closed, failure.

\section{Illustrative case study}

The proposed risk-informed monitoring approach was demonstrated on a simulacrum of a road bridge on the M6 motorway in the UK. It was assumed that the operator of the bridge is interested in knowing what benefit would they obtain by installing a bridge monitoring system. The LCC analysis was carried out for 50 years based on a different set of inspection and maintenance strategies adopted from (24) for informing Eqn. (2). For all strategies considered, it was assumed that minor repairs, major repairs and replacement will be carried out every $10^{\text {th }}, 20^{\text {th }}$ and $50^{\text {th }}$ year respectively. The frequency of servicing was delayed across different strategies. The associated unit costs are presented in Table 1. Different variables for estimating the operational impacts of bridge-related disruptions using Eqn. (3) was adopted from (25-30). Monte Carlo simulations using @Risk software was employed to deal with uncertainties associated with the variables. For illustrative purposes, we consider one of the strategies within the case study for further analysis. For the selected strategy, the maintenance costs were calculated to be $f 900,000$, the impact of bridge failure to be $£ 5,000,000$ and closure to be $£ 3,000,000$ at $90 \%$ confidence level. It was assumed that there is a $95 \%$ probability that a defect exists and the monitoring system has a $90 \%$ accuracy in detecting it.

Table 1. Variables used for estimating LCC (24)

\begin{tabular}{ccc}
\hline Variable & $\begin{array}{c}\text { Cost } \\
\text { (in } \mathbf{f} \text { ) }\end{array}$ & $\begin{array}{c}\text { Average } \\
\text { Disruption } \\
\text { (in days) }\end{array}$ \\
\hline Visual inspection & 2000 & \\
\hline Servicing & 3200 & 7,6 \\
\hline Minor repair & 5600 & 9,86 \\
\hline Major repair & 21300 & 118,64 \\
\hline Replacement & 80000 & 150 \\
\hline
\end{tabular}

The results from the LCC analysis was fed into the decision tree to inform if the bridge should be monitored or not. This was carried out using Precision Tree software that employs Monte Carlo simulations to deal with uncertainties associated with the variables at each node of Figure 2. To this end, the results with a $90 \%$ probability of occurrence (or confidence level) were obtained from 10,000 iterations and presented in Figure 3. The results show that the bridge in question can be considered for monitoring if a trade-off between the monitoring and LCC is achieved. It needs to be 
noted that these results would depend upon the ability of the monitoring system to detect the defect. E.g. the commonly employed visual inspection results in less accuracy, while continuous monitoring using sensors enables smarter and proactive asset management of bridges and increases the resilience and value of the transportation system.

\section{Discussion}

The continuous increase in the demand for transport networks creates a need for a riskinformed monitoring strategy, particularly for bridges, as they are critical elements within the transport infrastructure. To improve the safety and availability of the transport network within constrained budgets, innovative methods that can continuously monitor the bridges are required to provide rapid and reliable information to the decision-makers, to schedule current and future inspection and maintenance strategies

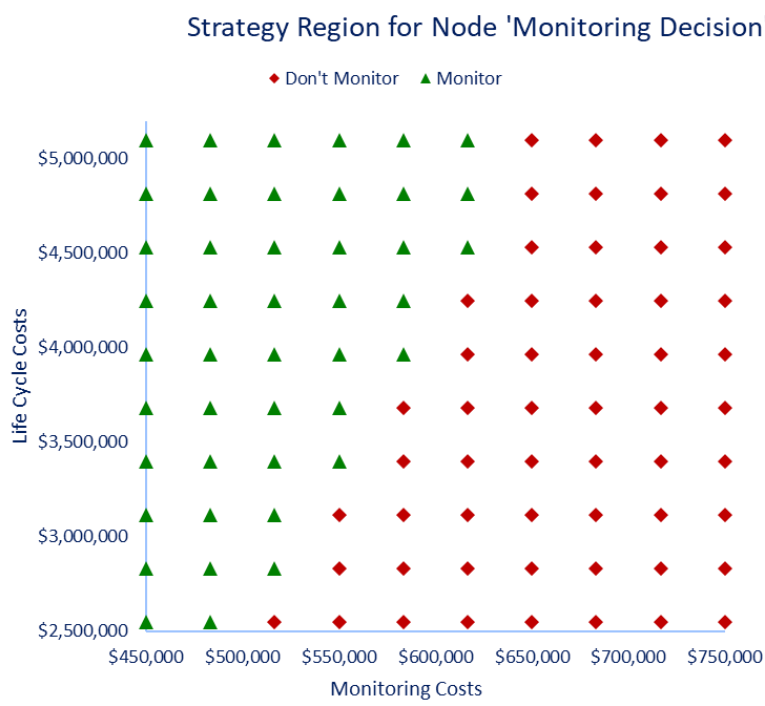

Figure 3 Decision making matrix for monitoring the bridge or not

Vol provides an important tool for choosing monitoring strategies. The proposed approach provides an efficient and practical bridge management framework that integrates $\mathrm{Vol}$ in the life cycle analysis. This offers the stakeholders transparent and effective management of cost, serviceability, the safety that can be applied when considering various types of inspection, monitoring and maintenance strategies Similarly, Vol can also be used for comparing different monitoring techniques and selecting the suitable one(s) for individual bridges. E.g. to use sonar for detecting scours with a higher probability of detection vs satellites that can provide more information about the structure and the surroundings. Since the accuracy of the monitoring system plays an important role in deciding if it is economicallyjustifiable, the exact properties of the system should be known and taken into consideration.

Considering that climate change is projected to increase the frequency and intensity of some extreme weather events, there is a need to extend current risk-informed monitoring systems to incorporate the effects of climate change, infrastructure interdependencies and early warning systems. The risks of individual bridges, as estimated within this paper, could also be amalgamated for an entire network or route, to yield a measure of overall bridge network performance.

\section{Conclusions}

The proposed approach is a simple approximation of the decision analysis that is ideally suited to be used in conjunction with life cycle analysis to determine whether a bridge should be monitored or not. The analysis in the paper did not focus on the exact bridge, element, or location; rather, used parameters representing a holistic situation of a general case. However, to perform the analysis, a particular bridge element or defect needs to be considered. Moreover, the decision tree presented within the analysis was stationary, meaning that the probabilities and costs of events were not associated with their time of occurrence. The timedependency of all the components needs to be taken into account to further investigate how the duration of certain events influences the business case for bridge monitoring. These are are being addressed through on-going research. Furthermore, the decision tree could be extended to include events such as wider economic impacts due to disruptions on services, the shift of rerouted traffic to other transport modes, unsuccessful reactive maintenance etc. 


\section{Acknowledgements}

The support from the Engineering and Physical Science Research Council (EPSRC) through the grant EP/N021614/1 (CSIC Innovation and Knowledge Centre Phase 2) and Innovate UK through the grant 920035 (Centre for Smart Infrastructure and Construction) are gratefully acknowledged.

\section{References}

[1] Vardanega P.J., Webb G.T., Fidler P.R.A., Middleton C.R. Assessing the potential value of bridge monitoring systems. Proceedings of the Institution of Civil Engineers: Bridge Engineering. 2016;169(2):126-38.

[2] Zitrou A., Bedford T., Daneshkhah A. Robustness of maintenance decisions: Uncertainty modelling and value of information. Reliability Engineering and System Safety. 2013;120:60-71.

[3] Malings C., Pozzi M. Value of information for spatially distributed systems: Application to sensor placement. Reliability Engineering and System Safety. 2016;154:219-33.

[4] Srinivasan R., Parlikad A.K. Value of condition monitoring in infrastructure maintenance. Computers and Industrial Engineering. 2013;66(2):233-41.

[5] Pozzi M., der Kiureghian A. Assessing the value of alternative bridge health monitoring systems. Bridge Maintenance, Safety, Management, Resilience and Sustainability - Proceedings of the Sixth International Conference on Bridge Maintenance, Safety and Management. 2012;782-9.

[6] Bisdikian C., Kaplan L.M., Srivastava M.B. On the quality and value of information in sensor networks. ACM Transactions on Sensor Networks. 2013;9(4).

[7] Zambon I, Ariza MPS, Matos JC, Strauss A. Value of information (Vol) for the chloride content in reinforced concrete bridges. Applied Sciences. 2020;10(2):1-16.

[8] Klerk W.J., Schweckendiek T., den Heijer F., Kok M. Value of information of structural health monitoring in asset management of flood defences. Infrastructures. 2019;4(3):1-20.

[9] Giordano P.F., Prendergast L.J., Limongelli M.P. A framework for assessing the value of information for health monitoring of scoured bridges. Journal of Civil Structural Health Monitoring. 2020;(May).

[10] Long L., Döhler M., Thöns S. Determination of structural and damage detection system influencing parameters on the value of information. Structural Health Monitoring. 2020;

[11] Giordano P.F., Prendergast L.J., Limongelli M.P. Impact of climate change on the Value of Information for bridges at risk of scour. In: 7th International Symposium on LifeCycle Civil Engineering, Shanghai, China. 2020.

[12] Bolognani D., Cappello C., Zonta D., Tonelli D., Verzobio A., Glisic B., et al. The conditional value of information of SHM: what if the manager is not the owner? Proc. SPIE 10600, Health Monitoring of Structural and Biological Systems XII 2018;87.

[13] Bennett J.R., Maxwell S.L., Martin A.E., Chadès I., Fahrig L., Gilbert B. When to monitor and when to act: Value of information theory for multiple management units and limited budgets. Journal of Applied Ecology. 2018;55(5):2102-13.

[14] HMEP. Highway infrastructure asset management. Department for Transport. 2013.

[15] Sasidharan M., Parlikad A.K., Schooling J. Risk-informed asset management to tackle scouring on bridges across transport networks. Structure and Infrastructure Engineering. 2021; 0(0):1-17

[16] Le B., Andrews J. Modelling railway bridge asset management. Proceedings of the 
Institution of Mechanical Engineers, Part F: Journal of Rail and Rapid Transit. 2013;227(6):644-56.

[17] Yianni P.C., Rama D., Neves L.C., Andrews J.D., Castlo D. A Petri-Net-based modelling approach to railway bridge asset management. Structure and Infrastructure Engineering. 2017;13(2):287-97.

[18] Rashidi M., Samali B., Sharafi P. A new model for bridge management: Part A: condition assessment and priority ranking of bridges. Australian Journal of Civil Engineering. 2016;14(1):35-45.

[19] Gong C., Frangopol D.M. Condition-Based Multiobjective Maintenance Decision Making for Highway Bridges Considering Risk Perceptions. Journal of Structural Engineering. 2020;146(5).

[20] Maroni A., Tubaldi E., Val D., Mcdonald H., Lothian S., Riches O., et al. A Decision Support System for Scour Management of Road and Railway Bridges Based on Bayesian Networks. In: The 12th International Workshop on Structural Health Monitoring, IWSHM 2019. 2019.

[21] Babanajad S., Bai Y., Wenzel H., Wenzel M., Parvardeh H., Rezvani A, et al. Life Cycle Assessment Framework for the U.S. Bridge Inventory. Transportation Research Record. 2018;2672(12):82-92.

[22] Zonta D., Gilsic B., Adriaenssens S. Value of information: impact of monitoring on decision-making. Structural Control and Health Monitoring. 2014;21:1043-56.

[23] Memarzadeh M., Pozzi M. Value of information in sequential decision making: Component inspection, permanent monitoring and system-level scheduling. Reliability Engineering and System Safety. 2016;154:137-51.

[24] Le B.L. Modelling railway bridge asset management using Petri-Net modelling techniques. PhD Thesis, University of Nottingham. 2014;

[25] Marsden G., Anable J., Chatterton T., Docherty I., Faulconbridge J., Murray L., et al. Studying disruptive events: Innovations in behaviour, opportunities for lower carbon transport policy? Transport Policy. 2020;94(April):89-101.

[26] Lamb R., Garside P., Pant R., Hall J.W. A Probabilistic Model of the Economic Risk to Britain's Railway Network from Bridge Scour During Floods. Risk Analysis. 2019;39(11):2457-78.

[27] Zhu S, Levinson D. A Review of Research on Planned and Unplanned Disruptions to Transportation Networks. Transportation Research Record. 2010;89.

[28] Department for Transport - DfT. Highways Economics Note No. 12005 Valuation of the Benefits of Prevention of Road Accidents and Casualties. 2007;(1):1-13.

[29] Department for Transport - DfT. Transport User Benefit Calculation. 2010;(TAG Unit 3.5.3).

[30] Department for Transport - DfT. Transport Appraisal And The New Green Book TAG Unit 2.7.1. 2004;(April). 